\title{
Leucoencefalopatía reversible posterior: cuatro casos clínicos
}

\author{
Marta Hernández $\mathrm{Ch}^{1}$, Alicia $\mathrm{N}$ úñez $\mathrm{F}^{1}$, Tomas Mesa $\mathrm{L}^{1}$, \\ Raúl Escobar $\mathbf{H}^{1}$, Francisco Barriga $\mathrm{C}^{2}$, Isidro H uete $\mathrm{L}^{3}$. \\ Reversible posterior \\ leukoencephalopathy syndrome in \\ patients with immunosupressive \\ treatment. Report of four cases
}

Reversible posterior leukoencephalopathy syndrome (PLS) is characterized by headache, clouding of sensorium, visual disturbances and seizures. It is associated to hypertension, renal disease or immunosuppressive therapy. We report three males, aged 9, 12 and 16 years and one female, aged 5 years wih PLS associated to immunosuppressive therapy. All had seizures and three had headache and clouding of sensorium. One case was associated to an hypertensive emergency, one to liver failure and one to high tacrolimus levels. Magnetic resonance imaging showed lesions in the white matter in two patients and in the gray matter in the other two. The lapse between the start of immunosuppressive treatment and neurological symptoms ranged from 4 days to 6 months. All received antiepileptic drugs and immunosuppresive therapy was changed or decreased, with complete clinical recovery (Rev Méd Chile 2008, 136: 93-8).

(Key words: Immunosupression; Posterior leukoencephalopathy syndrome; Tacrolimus)

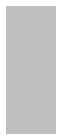

Recibido el 29 de agosto, 2006. Aceptado el 20 de abril, 2007.

Departamento de Pediatría, Sección Neurología Infantil ${ }^{1}$ y Hemato-oncología ${ }^{2}$, Departamento de Radiología ${ }^{3}$. Facultad de Medicina. Pontificia Universidad Católica de Chile. Santiago, Chile

$\mathrm{L}$ a "leucoencefalopatía reversible posterior" (LRP) es una encefalopatía de inicio agudo con convulsiones, cefalea, alteración del estado mental y trastornos visuales. Las neuroimágenes muestran compromiso de la sustancia blanca con predilección por lóbulos occipitales ${ }^{1}$.

Correspondencia a: Dra. Marta Hernández, Pontificia Universidad Católica de Chile. Facultad de Medicina. Departamento de Pediatría. Lira 81, Santiago Chile CP 6510273. Tel: 3546486. Fax 6384307.

E mail: mhernand@med.puc.cl
La patogenia es desconocida, pero se ha asociado a hipertensión arterial, eclampsia, enfermedades renales $^{2}$, terapia inmunosupresora (ciclosporina, tacrolimus e interferón-alfa) y quimioterapia ${ }^{3}$ (cisplatino, citarabina y metrotexato). Recientemente se ha incluido el síndrome hemolítico urémico, neoplasias, vasculitis, gammaglobulina endovenosa, quimioterapia, tratamiento con eritropoyetina y falla hepática aguda $^{4,5}$.

Si bien el 90\% de los pacientes con terapia inmunosupresora (TIS) cursan con hipertensión menos del 10\% presenta LRP, lo que sugiere que hay otros factores asociados. Se ha especulado que los inmunosupresores IS causan vasoconstric- 
ción, microembolismo y daño directo del endotelio vascular ${ }^{6}$ con trasudación hacia parénquima, lo que se exacerbaría con otros factores como hipocolesterolemia, hipomagnesemia, encefalopatía hepática o niveles plasmáticos elevados del inmunosupresor ${ }^{7}$. La disminución o cambio de éste parece atenuar la neurotoxicidad

En el cerebro, bajo condiciones de hipertensión, los nervios simpáticos perivasculares aumentan la resistencia vascular, para proteger el tejido cerebral. El sistema vertebrobasilar, responsable de la irrigación de estructuras de fosa posterior y lóbulos occipitales, posee poca inervación simpática, por lo que la vasoconstricción no logra inhibir la hiperperfusión produciéndose trasudación a territorio posterior ${ }^{8}$.

Actualmente, el término "leucoencefalopatía reversible posterior" ha sido objetado en sus tres elementos, se han descrito lesiones en sustancia gris $^{9}$, la evolución puede no ser reversible con evoluciones adversas y fatales ${ }^{10,11}$ hasta en el $26 \%$ de los casos y la localización puede ser fuera del territorio vertebrobasilar ${ }^{12,13}$.

El objetivo del presente trabajo es describir las características clínicas, de laboratorio, neuroimágenes, tratamiento y seguimiento de cuatro pacientes con LRP evaluados en este centro.

\section{Casos Clínicos}

Se presentan 4 pacientes con LRP que debutan con síntomas neurológicos agudos y alteraciones en la resonancia magnética (RM) cerebral entre enero 2002 y julio 2005.

Paciente 1. Niño, 9 años, con aplasia medular y trasplante de médula ósea (TMO). Inició TIS con ciclosporina (CS) presentando hacia el $4^{\circ}$ mes de tratamiento compromiso de conciencia y crisis tónico clónica hemigeneralizada precedida de aura visual. Al examen estaba normotenso, confuso, sin signos neurológicos focales. La función renal y hepática, magnesio, colesterol y electrolitos plasmáticos eran normales. Los cultivos y la búsqueda de anticuerpos contra virus y bacterias en sangre y líquido cefalorraquídeo (LCR) fueron negativos. Los niveles de CS eran de $216 \mathrm{ng} / \mathrm{mL}$ (nivel terapéutico hasta $300 \mathrm{ng} / \mathrm{mL}$ ) La RM cerebral informó lesiones simétricas de sustancia blanca occipital y parietal (Figura 1A y 1B). La difusión no mostró cambios sugerentes de isquemia aguda. El electroencefalograma (EEG) registró crisis temporales derechas subintrantes y disfunción lenta generalizada. Se trató con fenitoína endovenosa, y se cambió IS a tacrolimus. La sintomatología

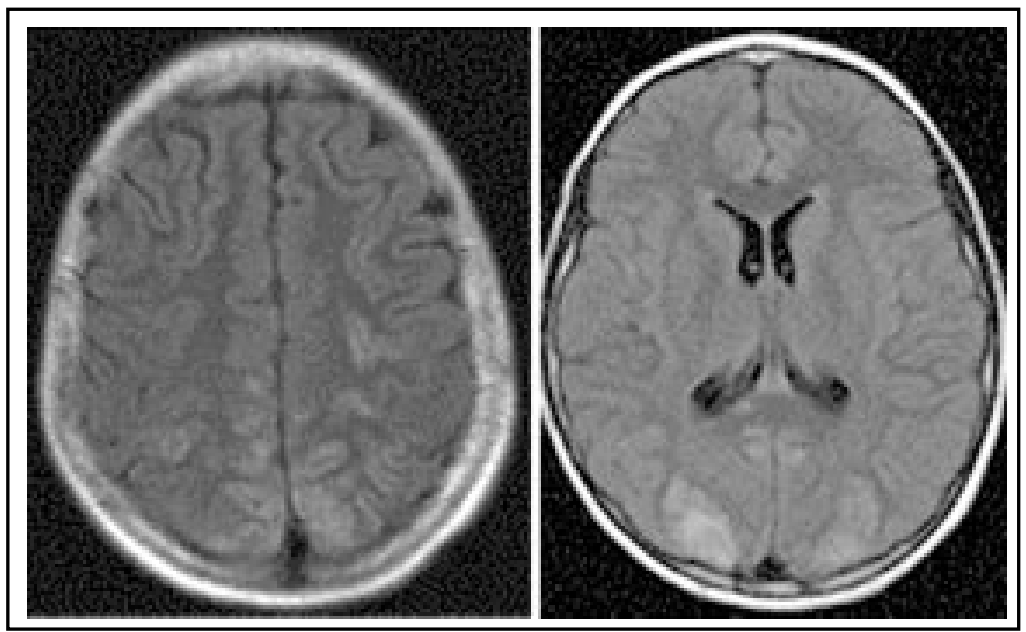

Figura 1. RM de cerebro, secuencia FLAIR que muestra hiperseñal frontal, parietal (1A) y occipitales bilaterales (1B) en periodo agudo. 
revirtió en el periodo de 2 semanas. El paciente se mantiene en control a 4 años del transplante, sin terapia antiepiléptica y en buenas condiciones.

Paciente 2. Niña con antecedentes de síndrome nefrótico corticoresistente desde los 2 1/2 años, con mala respuesta a ciclofosfamida. A los 5 años inició terapia con CS, 20 días después presentó cefalea intensa, crisis hipertensiva (155/113) compromiso de conciencia y nistagmus. El EEG informó status eléctrico subclínico. Cursó con hipokalemia, hipocalcemia, hipoalbuminemia e hipercolesterolemia (664 ug/dL), los cultivos y búsqueda de anticuerpos contra virus y bacterias en sangre, LCR y orina eran negativos, magnesemia normal, niveles de CS $169 \mathrm{ng} / \mathrm{mL}$. Se inició tratamiento de status epiléptico con fenitoina, fenobarbital y ácido valproico, tratamiento de crisis hipertensiva y suspensión de CS. La RM cerebral muestra lesiones hiperintensas en secuencias con TR largo en la corteza temporal mesial, cingulada y pulvinar del tálamo izquierdo. Su sintomatología cedió en el plazo de 3-4 semanas. El control de la RM cerebral en 2 meses muestra ausencia de lesiones (Figura 2). El tratamiento antiepiléptico se mantuvo por 1 año, sin recurrencias. La paciente estuvo en control por 3 años sin crisis y asintomática.

Paciente 3. Hombre, 16 años portador de Niemann Pick, con antecedentes de TMO previo. Ingresó para trasplante hepático. Se inició inmunosupresión endovenosa con tacrolimus, cuatro días después, presentó dos crisis tónico-clónica generalizada de corta duración. Examen neurológico normal intercrisis, excelente estado general, bien hidratado y bien perfundido. Los niveles de tacrolimus eran de 9,7 $\mathrm{ng} / \mathrm{mL}$ (máximo $15 \mathrm{ng} / \mathrm{mL}$ ), electrolitos, magnesio y colesterol normal. El EEG no mostró actividad epileptiforme. Se trató con fenitoína endovenosa. La RM cerebral informó focos de hiperseñal córticosubcortical en circunvoluciones frontal, media y parietooccipital (Figura 3). Se disminuyó transitoriamente dosis de tacrolimus. Se mantuvo terapia antiepiléptica con clobazam por dos meses. Seguimiento durante dos años sin recurrencia de crisis y asintomático neurológicamente.

Paciente 4. Hombre, 12 años, aplasia medular diagnosticada a los 8 años, tratado con ciclosporina y prednisona sin respuesta. Se realizó TMO e inició inmunosupresión con tacrolimus, seis meses después, ingresó a UCI por cefalea, compromiso de conciencia y convulsiones, normotenso. El colesterol, magnesio y electrolitos plasmáticos, estudio hepático y renal normales. Los cultivos y búsqueda de anticuerpos contra virus y bacterias en sangre y LCR eran negativos. El EEG intercrisis informó lentitud frontotemporal bilateral. Los niveles de tacrolimus se habían elevado a $32 \mathrm{ng} / \mathrm{mL} 48$ horas antes del episodio, disminuyendo a valores terapéuticos $(7,3 \mathrm{ng} / \mathrm{mL})$ el día de la convulsión. La RM cerebral en secuencias FLAIR (fluid attenuated inversion recovery) mostró áreas de hiperseñal

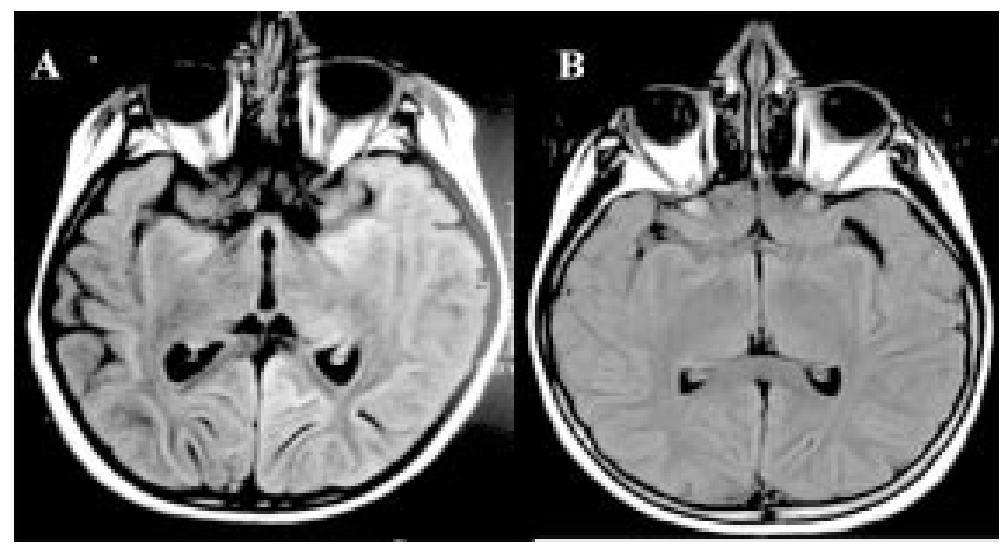

Figura 2: A. RM de cerebro, secuencia FLAIR, nuestra alteraciones de la corteza temporal mesial, cingulada y pulvinar del tálamo izquierdo B. RM control a los dos meses, secuencia FLAIR, con regresión total de las alteraciones. 
confluentes cortico subcorticales en lóbulos frontales, temporales y occipitales bilaterales. Se disminuyó la dosis de tacrolimus y se trató con fenitoina por 12 meses. Se recuperó progresivamente, sin déficit neurológico posterior y sin nuevas crisis. El control de RNM a los dos meses mostró franca regresión de las lesiones (Figura 4). Seguimiento a 1 año sin recaída de encefalopatía.

\section{DiSCUSIÓN}

La aparición de sintomatología neurológica aguda en un paciente con TIS debe alertar, entre otros diagnósticos, a neurotoxicidad. La neurotoxicidad puede ser mayor o menor. La neurotoxicidad menor ${ }^{14}$ aparece durante la carga endovenosa hasta en $30 \%$ de los pacientes y se manifiesta como cefalea, temblor, parestesias e insomnio. La neurotoxicidad mayor ${ }^{15,16}$ aparece después de los 14 días y se manifiesta con convulsiones, mutismo akinético, polineuropatía, miopatía y en su expresión mayor la LRP. La ciclosporina y el tacrolimus son IS inhibidores de la calcineurina ${ }^{17}$, enzima clave en la respuesta aloinmune específica, a diferencia del sirolimus y micofenolato, que no actúan mediante este mecanismo y no presentan neurotoxicidad ${ }^{18}$.

En la literatura se reporta un tiempo de latencia entre el inicio de la TIS y la aparición de

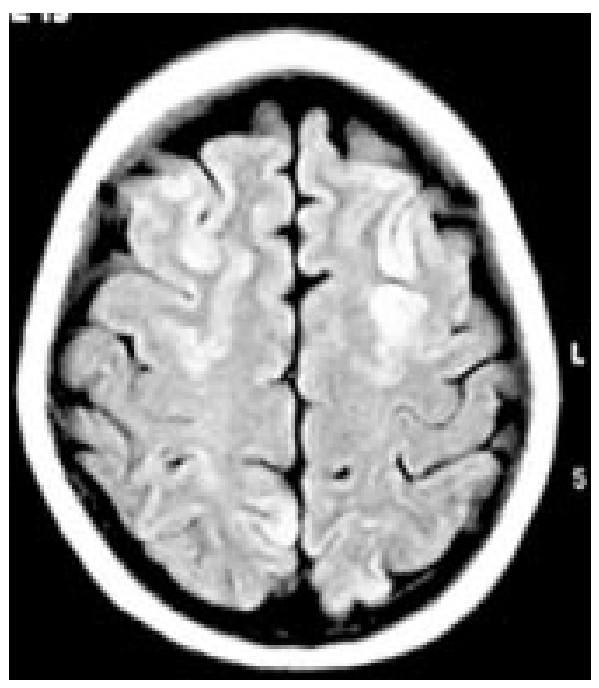

Figura 3: RM, secuencia FLAIR, muestra múltiples focos de hiperseñal córticosubcortical bilaterales en circunvoluciones frontales medias y parietooccipitales.

signología neurológica de 4 días a 5 años ${ }^{19}$. En nuestros pacientes tuvimos un caso de aparición precoz (menos de 4 días), que correspondería a neurotoxicidad mayor a pesar de su precocidad, con rápida mejoría de sus síntomas (caso 3) y 3 casos de aparición tardía, con un cuadro clínico

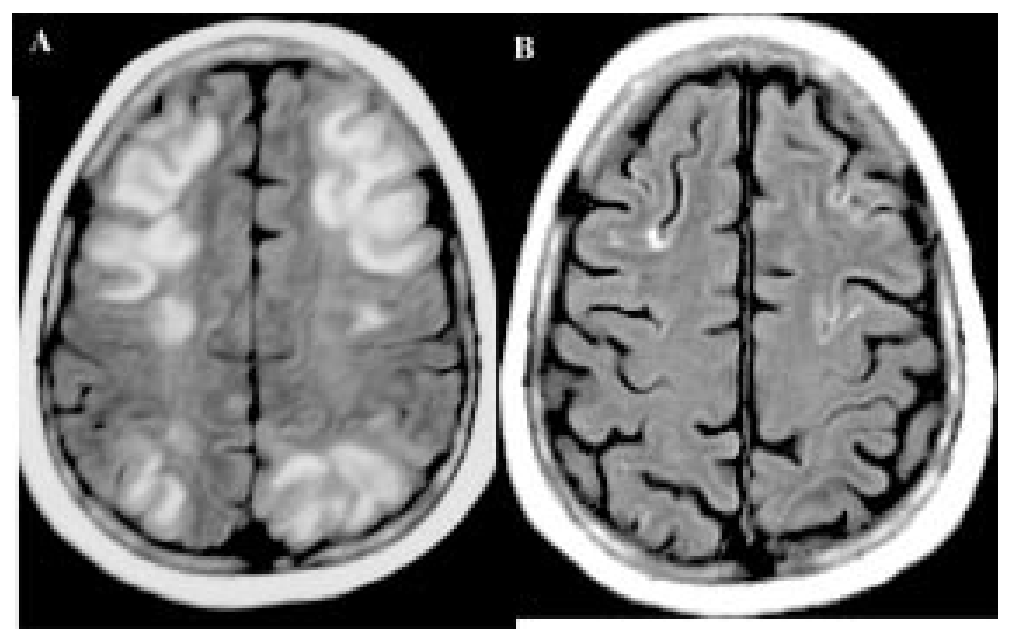

Figura 4: A. RM secuencia FLAIR, muestra áreas confluentes de hiperseñal cortico subcortical en lóbulos frontales y parietales bilaterales. B. RM de control a los 2 meses, secuencia FLAIR, muestra franca regresión de las alteraciones. 
más grave y de más lenta recuperación (caso 1, 2 y 4). Todos nuestros pacientes debutaron con convulsiones, las que fueron generalizadas en dos pacientes, status epiléptico no convulsivo en uno y crisis parcial con aura visual en otro (Tabla 1).

Aun cuando no se ha encontrado relación absoluta entre los niveles séricos de IS y severidad de los síntomas neurológicos e incluso se describen niveles plasmáticos terapéuticos hasta en 50\% de los casos, la reducción de la dosis o el cambio transitorio de IS se ha asociado con mejoría clínica $^{20}$. En nuestros pacientes, solo uno tuvo niveles supraterapéuticos de IS. El resto tenía niveles adecuados pero tenían otros factores predisponentes como crisis hipertensiva severa, hipoalbuminemia y disfunción hepática. En ninguno obtuvimos el antecedente de uso de metoclopramida, que se describe como una interacción farmacológica precipitante de neurotoxicidad ${ }^{21}$.

Desde el punto de vista de neuroimágenes, sólo en dos casos encontramos compromiso exclusivo de sustancia blanca, pero sin predilección poste- rior, contrario a lo que clásicamente se describe por la menor inervación simpática del territorio vertebrobasilar. En los dos casos restantes el compromiso fue predominantemente cortical, lo que retrasó el diagnóstico, pero la ausencia de lesiones en secuencia de difusión, excluyó la posibilidad de focos de isquemia cortical (Figuras 1 y 3). La difusión (Diw) y los mapas de coeficiente de difusión (ADC) en la LRP son normales o muestran áreas con difusión aumentada, lo que sugiere neurotoxicidad más que isquemia aguda (difusión restringida ${ }^{22}$. Las alteraciones de sustancia blanca son mejor visualizadas en la secuencia FLAIR.

Las manifestaciones neurológicas agudas observadas en estos pacientes podrían haber correspondido a una variedad de patologías como accidentes vasculares, trombosis de senos venosos, encefalomielitis aguda diseminada, encefalitis, encefalopatía anóxica, mielinolisis pontina central o extracentral y encefalopatía hipoglicémica, las que tienen un tratamiento y pronóstico diferente. Sólo la RM cerebral permite diferenciarlas y debe realizarse precozmente

Tabla 1. Características clínicos de pacientes con LRP

\begin{tabular}{|c|c|c|c|c|c|}
\hline Caso & $\begin{array}{l}\text { Edad (a) } \\
\text { Sexo }\end{array}$ & Morbilidad & Inmunosupresor & clínica & PA \\
\hline 1 & $9 / \mathrm{M}$ & TMO, aplasia medular & ciclosporina & $\begin{array}{l}\text { Sopor, cefalea, fotopsias, } \\
\text { convulsión parcial }\end{array}$ & $130 / 85$ \\
\hline 2 & $4 / \mathrm{F}$ & $\begin{array}{l}\text { Síndrome nefrótico } \\
\text { córtico resistente }\end{array}$ & ciclosporina & $\begin{array}{l}\text { Confusión, afasia, estado } \\
\text { epiléptico parcial complejo. }\end{array}$ & $155 / 113$ \\
\hline 3 & $16 / \mathrm{M}$ & $\begin{array}{l}\text { Trasplante hepático } \\
\text { (Nieman Pick) }\end{array}$ & tacrolimus & Convulsiones generalizadas & $140 / 90$ \\
\hline 4 & $12 / \mathrm{M}$ & TMO, aplasia medular & tacrolimus & $\begin{array}{l}\text { Cefalea, sopor, convulsiones, } \\
\text { hemiparesia }\end{array}$ & $140 / 86$ \\
\hline
\end{tabular}

Tabla 2. H allazgos radiológicos y electroencefalográficos de pacientes con LRP

\begin{tabular}{|lll|}
\hline Pac RNM cerebral & EEG \\
\hline 1 & $\begin{array}{l}\text { Hiperseñal subcortical lóbulos occipitales, parietales } \\
\text { y frontales }\end{array}$ & Crisis eléctricas temporales derechas subintrantes \\
2 & $\begin{array}{l}\text { Aalteraciones de la corteza temporal mesial, cingulada } \\
\text { y pulvinar del tálamo izquierdo }\end{array}$ & $\begin{array}{l}\text { Lentitud rítmica hemisférica izquierda, } \\
\text { sugiere status subclínico }\end{array}$ \\
3 & $\begin{array}{l}\text { Múltiples focos de hiperseñal córticosubcortical bilaterales } \\
\text { en circunvoluciones frontales medias y parietooccipitales, }\end{array}$ & Sin actividad epileptiforme. \\
4 & $\begin{array}{l}\text { Áreas confluentes de hiperseñal cortico subcortical en } \\
\text { lóbulos frontales y parietales bilaterales }\end{array}$ & Lentitud frontotemporal bilateral \\
\hline
\end{tabular}


analizando las secuencias FLAIR y Diw. Las formas atípicas de LRP son muy difíciles de distinguir de otras enfermedades por lo que el índice de sospecha debe ser alto. La prevención requiere un TIS en una dosis mínima eficaz, administración oral, tan pronto como sea posible, monitorización estricta de niveles plasmáticos (incluyendo metabolitos), balance electrolítico (incluyendo magnesio), mantener normotensión y evitar las interacciones farmacológicas.

\section{REFERENCIAS}

1. Hinchey J, Chaves C, Appignani B, Breen J, Pao L, Wang A ET AL. A reversible posterior leukoencephalopathy syndrome. N Engl J Med 1996; 334: 494-500.

2. Giménez-Mesa E, Martínez-Salio A, Porta-Etessam J, Berbel A, Cedena T, Salama P. Leucoencefalopatía posterior reversible en un paciente con linfoma noHodgkin tras tratamiento con CHOP. An Med Interna 2001; 18: 591-3.

3. Walker RW, Allen JC, Rosen G, Caparros B. Transient cerebral dysfunction secondary to high-dose methotrexate. J Clin Oncol 1986; 4: 1845-50.

4. Kwon S, Koo J, LeE S. Clinical spectrum of reversible posterior leukoencephalopathy syndrome. Pediatr Neurol 2001; 24: 361-4.

5. Lanzino G, Cloft H, Hemstreet Mk, West K, Aiston S, IsHITAN M. Reversible posterior leukoencephalopathy following organ transplantation: description of two cases. Clin Neurol Neurosurg 1997; 99: 222-6.

6. Inoha S, Inamura T, NaKamizo A, Ikezaki K, Amano T, Fuku M. Magnetic resonance imaging in cases with encephalopathy secondary to immunosuppressive agents. J Clin Neurosc 2002; 9: 305-7.

7. Port JD, Beauchamp NJ. Reversible intracerebral pathologic entities mediated by vascular autoregulatory dysfunction. Radiographics 1998; 18: 353-67.

8. López-García F, Espinosa-Garriga G. Síndrome de leucoencefalopatía posterior reversible. Rev Clin Esp 2000; 200: 57-9.

9. Koch S, Rabinstein A, Falcone S, Forteza A. Diffusionweighted imaging shows cytotoxic and vasogenic edema in eclampsia. Am J Neuroradiol 2001; 22: 1068-70.

10. Ay H, Buonanno FS, Schaefer PW, Le DA, Wang B, Gonzalez RG, Koroshetz WJ. Posterior leukoencephalopathy without severe hypertension: utility of diffusion-weighted MRI. Neurology 1998; 51: 1369-76.

11. Covarrubias DJ, Luetmer PH, Campeau NG. Posterior reversible encephalopathy syndrome: prognostic uti-
Finalmente otras drogas inmunosupresoras como sirolimus y micofenolato no inhiben la calcineurina y no se han asociado a neurotoxicidad.

\section{Agradecimientos}

Agradecemos al Dr Jorge Martínez Castillo, Profesor Auxiliar de Cirugía, Pontificia Universidad Católica de Chile, la posibilidad de compartir y estudiar en conjunto a un paciente.

lity of quantitative diffusion-weighted MR images. Am J Neuroradiol 2002; 23: 1038-48.

12. Bianco F. Reversible posterior leukoencephalopathy syndrome: a changing concept. Neuroradiology 2005; 47: 703-4.

13. Casey SO, Sampaio RC, Michel E, Truwit Cl. Posterior reversible encephalopathy syndrome: utility of fluidattenuated inversion recovery MR imaging in the detection of cortical and subcortical lesions. Am J Neuroradiol 2000; 21: 1199-1206.

14. Guarino M, Stracciari A, D'Aiessandro R. A prospective study on the neurological complications after liver transplantation. Gastroenterology International 1999; 12: $140-5$.

15. Wijdicks EF, Wiesner RH, DahlKe LJ, Krom RA. FK506induced neurotoxicity in liver transplantation. Ann Neurol 1994; 35: 498-501.

16. KROUWER HG, WIJDICKS EF. Neurologic complications of bone marrow transplantation. Neurol Clin N Am 2003; 21: 319-52.

17. Fuentes JJ, Genesca L, Kingsbury TJ, Cunningham KW, Pérez-Riba M, Estrvill X et al. DSCR1, overexpressed in Down syndrome, is an inhibitor of calcineurin mediated signaling pathways. Hum Mol Genet 2000; 9: $1681-90$.

18. Kniepeiss D, Iberer F, Grasser B, Schaffellner $S$, TschelessnigG KH. Sirolimus and mycophenolate mofetil after liver transplantation. Transplant International 2003; 16: 504-9.

19. Schwartz R, Bravo Sm, Klufas Ra, Hsu l, Barnes Pd, RoBson CD ET AL. Cyclosporine neurotoxicity and its relationship to hypertensive encephalopathy: CT and MR findings in 16 cases. AJR 1995; 165: 627-31.

20. Schudring J, Wesseling P, VerRirs A. Severe tacrolimus leukoencephalopathy after liver transplantation. Am J Neuroradiol 2003; 24: 2085-8.

21. Guarino M, Bentto-León J, Decruyenafre J, Schmutzhard E, Weisseborn K, Stracciari A. EFNS guidelines on management of neurological problems in liver transplantation. Eur J Neurol 2006; 13: 2-9. 